\title{
Dielectric relaxation analysis of hybrid Acrylic-Polyurethane gels
}

\author{
Gerardo Martínez-Rugerio $^{a}$, Ángel Alegría ${ }^{a, b}$, Arantxa Arbe $^{a}$, Vesna \\ Daniloskac ${ }^{c}$ Juan Colmenero ${ }^{\mathrm{a}, \mathrm{b}, \mathrm{d}}$ \\ ${ }^{a}$ Centro de Física de Materiales (CSIC-UPV/EHU)-Materials Physics Centre (MPC), \\ Paseo Manuel Lardizabal 5, 20018, San Sebastián, Spain. \\ ${ }^{b}$ Departamento de Física de Materiales, Universidad del País Vasco (UPV/EHU) \\ Apartado 1072, 20080, San Sebastián, Spain. \\ ${ }^{c}$ Polymat and Departamento de Química Aplicada, Facultad de Ciencias Químicas, \\ University of the Basque Country UPV/EHU, Jose Mari Korta Zentroa, Tolosa \\ Hiribidea 72, Donostia / San Sebastian, Spain. \\ ${ }^{d}$ Donostia International Physics Centre, Paseo Manuel Lardizabal 4, 20018, San \\ Sebastian, Spain.
}

\begin{abstract}
In this paper we present a dielectric relaxation analysis of three AcrylicPolyurethane gel samples in connection with their synthesis conditions. The dielectric analysis was carried out using Broadband Dielectric Spectroscopy (BDS). Additionally, Differential Scanning Calorimetry (DSC) and Small Angle X-ray Scattering (SAXS) measurements were performed. The thermodynamic characterization outcomes evidence very similar characteristics of the glass transition phenomena among the investigated samples without any signature of melting or crystallization. X-ray diffraction patterns evidence distinctive features for the three gel samples in two different regions, i.e., at high and low values of the scattering vector q. BDS measurements, identify three distinct dielectric relaxation processes above Tg. Particularly, differences in the dielectric parameters of the slowest relaxation process have been rationalized in connection with the synthesis method used. Our results show that the analysis of these Acrylic-Polyurethane gels by BDS -in combination with SAXS- provides access to characteristics of the network structure that would ultimately influence the properties of these materials.
\end{abstract}

Keywords: Dielectric Relaxation, Acrylic-Polyurethane gels, X-ray diffraction. 


\section{Introduction}

Aqueous polyurethane (PU) dispersions are widely used for applications such as adhesive and coatings. In the adhesive industry the achievement of materials with good properties is a first prerequisite for further technological applications $[1,2,3]$. In order to obtain desired PU dispersions with improved properties, such as mechanical stability, toughness, solvent and chemical resistance, and to reduce costs, mixtures with poly-acrylic systems are used. Blending a poly-acrylic dispersion into a polyurethane dispersion often leads to final products with low quality, attributed to the poor compatibility between their components. However, the formation of hybrid particles where each particle contains both polymers and chemical linkages between them leads to a better compatibility of the components. Therefore, miniemulsion polymerization $[4,5,6]$ is a suitable technique to synthesize complex materials that cannot be produced otherwise. Furthermore, miniemulsion photopolymerization technique allows obtaining different good quality materials by varying the initial synthesis conditions. In the literature we can find several comprehensive reports on the fabrication and chemical characterization of Acrylic-PU dispersions [5, 7, 8], in particular of pressure sensitive adhesives (PSA's). One of the main conclusions obtained from these reports is that the adhesive properties of PSA's materials are mostly determined by their polymer architecture [9]. Therefore, understanding the polymer architecture is of great importance for synthesizing optimal tailored adhesives. By studying the structural and dynamical properties of three different latexes synthesized with the same components but with different synthesis conditions, we intend to get a deeper insight on the relation between the synthesis conditions and the corresponding material properties, for this a molecular description of the polymer architecture of the produced latexes is required.

Making use of broadband dielectric spectroscopy (BDS) technique we have already reported a suitable molecular interpretation for the dielectric relaxation phenomena in the gel and sol fractions of a characteristic latex obtained via miniemulsion photo-polymerization [10]. With this information at hand, in this work we study three distinct gel fractions corresponding to hybrid Acrylic-Polyurethane latexes synthesized with different initial conditions. The objective of this study is to obtain, compare and understand, different molecular characteristics of these gel networks as inferred by BDS. The results are complemented with structural characterization of the distinct gel fractions given by Small Angle X-ray Scattering. 


\section{Experimental}

A complete description of the materials and the synthesis methods, employed in the production of the studied hybrid Acrylic-PU pressure sensitive adhesives (PSA's) is described elsewhere [5, 11, 12, 13]. In brief, first the organic phase was prepared by dissolving the Incorez 701 (10 weight based on organic phase \%, 10 wbo\%), Bisphenol A (BPA) chain extender and DBTDL catalyst (550 ppm based on the organic phase) in the monomer mixture (2ethylhexylacrylate (2EHA)/n-octadecylacrylate (SA)/methyl methacrylate (MMA)/methacrylic Acid (MAA)/hydroxyethylmethacrylate (HEMA): 91.5 / 5.8 / 1.4 / 0.9 / 0.4 weight $\%$ ). Then, the organic phase was mixed with an aqueous solution of the surfactant (Downfax 2A1, 2\% wbo) and $\mathrm{NaHCO}_{3}$ $(0.02 \mathrm{M})$ under intensive stirring $(15 \mathrm{~min}$ at $1000 \mathrm{rpm})$ to create an emulsion. The resulting coarse emulsion was sonicated for 15 min at 9 output control and $80 \%$ duty cycle with a Branson 450 (Danbury, CT). Finally, in order to improve the miniemulsion stability, SDS was added (1\% wbo).

The polymerizations were performed in two different ways, either by photo-polymerization or by thermal polymerization. In the case of the photopolymerization, the photoinitiator (non-bleaching oil soluble photo initiator 1-hydroxycyclohexyl phenyl ketone, I-184 or 1-[4-(2-Hydroxyethoxy)phenyl]-2-hydroxy-2-methyl-1-propane-1-one, I-2959) were added to the organic phase before preparing the miniemulsion. The miniemulsion was then photopolimerized in a continuous quartz tubular reactor placed in a UV chamber (model BS 03, Dr. Grbel UV-Elektronik $\mathrm{GmbH}$ ) at $25^{\circ} \mathrm{C}$ with different incident light irradiances. For the sample prepared via thermal polymerization the miniemulsion was polymerized by a semi-continuous process in a $750 \mathrm{ml}$ glass-jacketed reactor equipped with a reflux condenser, sampling device, $\mathrm{N}_{2}$ inlet, two feeding inlets, and a stainless steel anchor-type stirrer rotating at $250 \mathrm{rpm}$. Initially, $20 \mathrm{wt} \%$ of the miniemulsion was placed into the reactor and allowed to polymerize over 1 hour by adding $0.48 \mathrm{wt} \%$ (weight based on monomer weight) initiator (Potassium persulfate, KPS). The rest of the miniemulsion and the initiator solution were then fed for 3 hours. After the end of the feeding period, polymerization was allowed to occur for 2 hours. The reaction temperature was $80^{\circ} \mathrm{C}$.

Regardless which miniemulsion process is used as a synthesis method, the resulting products are always latexes composed by a gel fraction and a sol fraction that coexist within each miniemulsion droplet. In order to separate both parts of the latex we used Soxhlet extraction under THF reflux. 
Table 1 summarizes relevant information on these materials such as: gel percentage (gel \%) photo-initiator type (PI), photo initiator concentration (PI \%) and incident light irradiance (ILI).

Table 1: Synthesis conditions for three Acrylic-Polyurethane latex, only gel fraction was studied

\begin{tabular}{|c|c|c|}
\hline Latex Samples & gel $\%$ & Synthesis Conditions \\
\hline A & 73 & $0.75 \%$ PI $(\mathrm{I}-2959), 7\left(\mathrm{~mW} / \mathrm{cm}^{2}\right)$ \\
\hline B & 46 & $0.25 \%$ PI (I-184), $3.5\left(\mathrm{~mW} / \mathrm{cm}^{2}\right)$ \\
\hline C & 67 & Thermal $\left(80^{\circ}\right)$ \\
\hline
\end{tabular}

The concentration and type of photo initiator used, as well as the amount of incident light irradiance used in the fabrication of the studied samples have the optimum values for synthesizing hybrid PSAs with good standards for adhesive properties. When preparing the samples for different experimental setups used in this work, all gel fractions were stored at $80^{\circ} \mathrm{C}$ in a vacuum oven for twelve hours. After this period of time the samples were allowed to cool down to room temperature in the vacuum oven before placing them into the corresponding sample holders. Before the experiments were performed, in situ heating up to $100^{\circ} \mathrm{C}$ of the samples was conducted to remove any trace of moisture.

\section{Techniques}

\subsection{Differential Scanning Calorimetry (DSC)}

Differential scanning calorimetric experiments were carried out using a Q2000 set up from TA Instruments with a liquid nitrogen cooling system. Temperature-modulated experiments were conducted using $60 \mathrm{~s}$ period and $0.5 \mathrm{~K}$ in amplitude, during cooling and heating at a mean rate of $3 \mathrm{~K} / \mathrm{min}$, in the temperature range 150 to $420 \mathrm{~K}$. Samples for DSC experiments were prepared by encapsulating 5-10 mg of sample in aluminum pans.

\subsection{Broadband Dielectric Spectroscopy (BDS)}

Measurements of the frequency dependent complex dielectric permittivity $\left(\epsilon^{*}(f)=\epsilon^{\prime}(f)-i \epsilon^{\prime \prime}(f)\right)$ were performed in the range $\mathrm{f}: 10^{-1}-10^{6} \mathrm{~Hz}$, using a Novocontrol high-resolution dielectric analyzer. The sample capacitor preparation for this setup consisted in an upper gold-coated electrode of $20 \mathrm{~mm}$ 
placed on a prepared film of the sample over a $30 \mathrm{~mm}$ gold-coated electrode. The sample cell was set in a cryostat, and its temperature was controlled via nitrogen gas jet stream coupled with the Novocontrol Quatro controller. The dielectric experiments were performed isothermally while cooling the sample from 420 to $150 \mathrm{~K}$ in steps of $5-10 \mathrm{~K}$. The data reproducibility was checked during subsequent heating from 150 to $410 \mathrm{~K}$.

\subsection{Small Angle X-Ray Scattering (SAXS)}

SAXS experiments were performed by means of Rigaku PSAXS-L equipment operating at $45 \mathrm{KV}$ and $0.88 \mathrm{~mA}$. The MicroMax-002 X-ray generator system is composed by a micro focus sealed tube source module and an integrated X-ray generator, which produces $\mathrm{Cu} \mathrm{K} \mathrm{K}_{\alpha}$ transition photons with wavelength of $\lambda=1.54 \AA$. The flight path and the sample chamber in this equipment are under vacuum. The scattered X-rays are detected on a twodimensional multi-wire detector (2D-200X) with $200 \mathrm{~mm}$ diameter active area with ca. 200 micron resolution and converted to one-dimensional scattering curves by radial averaging. The scattered intensities are presented as a function of the scattering vector $\mathrm{q}, \mathrm{q}=4 \pi(\lambda)^{-1} \sin (\theta)$, where $\theta$ is half the scattering angle. The sample to detector distance was set to $2 \mathrm{~m} \& 50 \mathrm{~cm}$ \& $20 \mathrm{~cm}$, covering a q range from 0.008 to $1.7 \AA^{-1}$. In this way, the range usually considered as WAXS (local length scales) was also explored.

\section{Results}

\subsection{Differential Scanning Calorimetry}

Figure 1 shows the reversing heat capacity as a function of the temperature for the three gel samples under study, the data were obtained while cooling the materials from $420 \mathrm{~K}$ at a rate of $3 \mathrm{~K} / \mathrm{min}$. The DSC data showed no signatures of crystallization and/or melting processes in the explored temperature range (up to $470 \mathrm{~K}$ ). Moreover, the three samples exhibit similar thermal behavior, particularly in the glass transition region, where all present a clear step like feature at a temperature of about $210 \mathrm{~K}$. In order

to highlight possible differences (if present) we also used the derivative of the reversible heat capacity as a more sensitive representation (depicted as inset in Figure 1). In the inset, we can notice a distinguishable peak centered around $210 \mathrm{~K}$ that corresponds to the glass transition which is similar for the three samples. Furthermore, the thermodynamic curves present similar behavior up to temperatures of about $420 \mathrm{~K}$, within the statistical errors. 


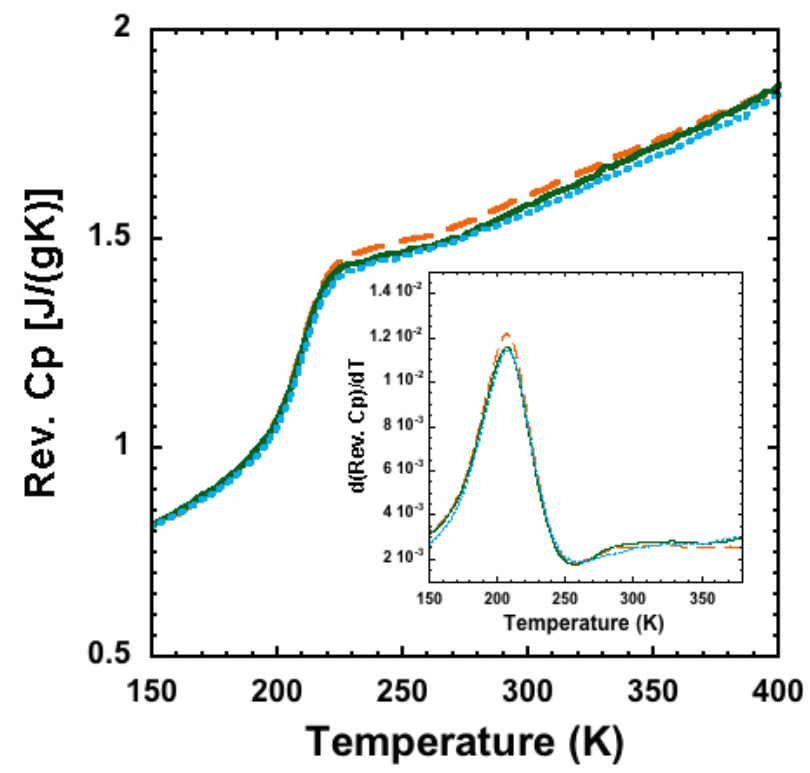

Figure 1: Differential Scanning Calorimetry curves for samples Gel A (dashed line), Gel B (solid line) and Gel C (dotted line). The inset shows the reversible heat capacity derivative with respect to temperature for all samples.

Thus, even though DSC is a useful technique and help us to identify the glass transition temperature for these particular Acrylic-Polyurethane samples (at about $210 \mathrm{~K}$ ), it does not reveal any clear difference related with the synthesis conditions.

\subsection{Broadband Dielectric Spectroscopy}

Figure 2a presents the real part of the dielectric permittivity as a function of the temperature at a frequency of $1 \mathrm{~Hz}$ (isochronal representation). This way of presenting the data allow us to visually identify the relaxation phenomena occurring in different temperature regions. Here we can clearly distinguish three different regions where dielectric processes occur. In a previous publication, the dielectric relaxation of the Gel B sample in comparison to both, that of the sol fraction and that of a purely acrylic sample, was reported. In this way, we were able to identify three different molecular entities responsible for the dielectric relaxation phenomena observed in the gel fraction. The dielectric relaxation phenomenon present at low temperatures (about $210 \mathrm{~K}$ ) was related to the segmental motion of dipole groups corresponding to the acrylic backbone, that is the $\alpha$-relaxation $(\alpha \mathrm{R})$. The 
relaxation process observed at about $280 \mathrm{~K}$ was related to motions involving Polyurethane (PU) chains attached to polymer acrylic backbones by only one end, since it was the most prominent in the sol fraction. This relaxation was referred to as dangling chain relaxation (DCR). The relaxation process observed at temperatures above $320 \mathrm{~K}$ was only present in the gel fraction of the previously studied latex and therefore it is closely related to the network structure. This relaxation phenomenon was attributed to dipolar entities formed by aggregation into bundles of PU chains which are responsible for the reticulated structure of the gel and for this reason named network relaxation (NR).

When comparing the real part of the dielectric data of different gel samples (see Figure 2a), we notice that in the lowest temperature region (about $210 \mathrm{~K}$ ) the real part of the dielectric permittivity exhibits a similar welldefined step present in the three samples, this similarity is in accordance with the coincident glass transition observed by means of DSC on the samples. Figure $2 \mathrm{~b}$ presents the imaginary part of the dielectric permittivity in the isochronal representation at the frequency of $1 \mathrm{~Hz}$. The dielectric data in the first temperature region also confirm these results. Consequently, as this relaxation is attributed to the movement of dipolar segments of the polymeric acrylic backbones and it is not affected by the synthesis method the $\alpha$-relaxation will not be the further discussed in this work.

Looking at higher temperatures of about $240-300 \mathrm{~K}$, in Figure 2a we can notice a second relaxation process visible for Gel A and Gel C samples and hardly identifiable in this representation for Gel B. As above commented, this relaxation process was related to the dangling chain dynamics of the $\mathrm{PU}$ polymers attached to the acrylic backbone. In this high temperature range the dc conductivity contributions to the dielectric losses strongly overlap the dipolar relaxation (see Figure 2b). Nevertheless, between $240 \mathrm{~K}$ and $300 \mathrm{~K}$, in the loss permittivity we can still detect the presence of the DCR relaxation process, namely for Gel A and Gel C, and not so obvious for Gel B.

At temperature above $320 \mathrm{~K}$, in Figure 2a we detect a third relaxation process visible in the three gel samples, that would be the most relevant in connection with the network characteristics. Observing the dielectric data in this temperature range we notice that the jump of the permittivity in this relaxation is quite small in the case of $\mathrm{Gel} \mathrm{C}$. This relaxation is completely hindered in the dielectric loss data.

In order to extract more information about the network-related phenomena a quantitative evaluation is required. Hence we analyzed the data in 


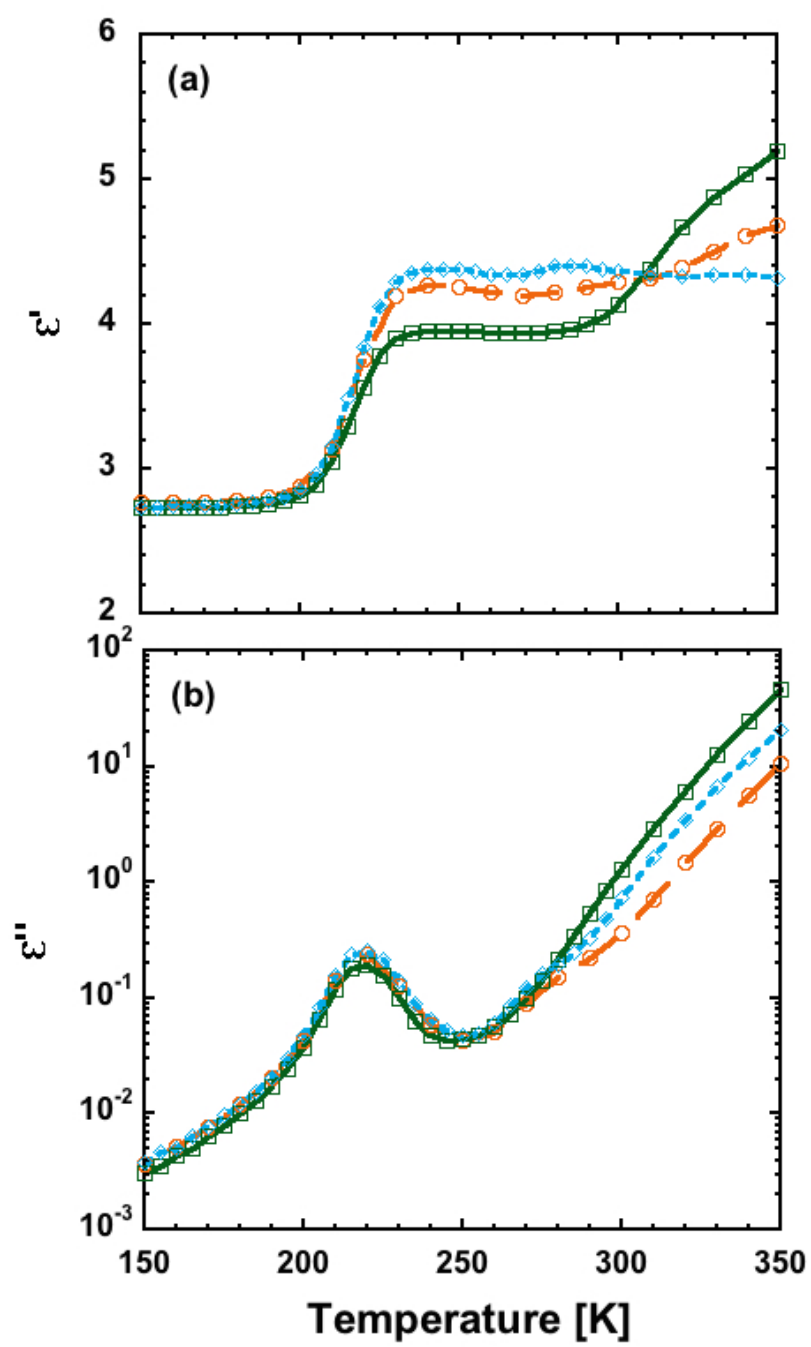

Figure 2: Real (a) and Imaginary (b) parts of the dielectric permittivity as a function of temperature for Gel A (circles), Gel B (squares), and Gel C (diamonds), in the isochronal representation at $1 \mathrm{~Hz}$. Lines in the figure are used as guides for the eye.

the isothermal representation. Due to the strong conductivity contributions at high temperatures, the analysis of the dielectric relaxation behavior was performed using the first derivative of the real part of the dielectric permittivity $[14,15]$.

Figure 3 presents the dielectric data in the derivative representation at a temperature of $420 \mathrm{~K}$ for the three gels. In this representation, a step-like 


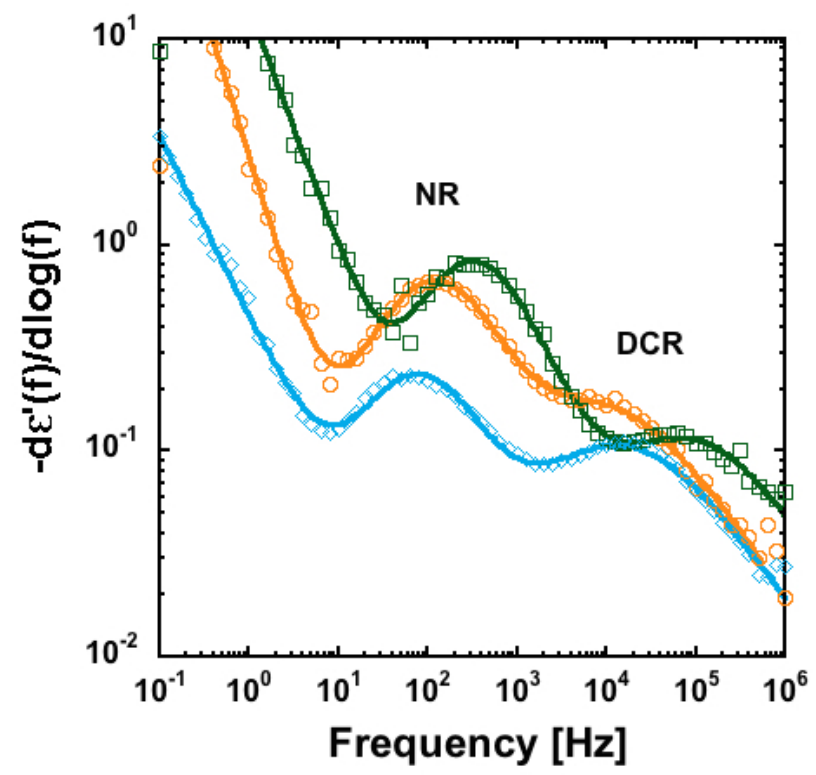

Figure 3: Derivative of the real part of the dielectric function with respect to the logarithm of frequency for the three samples Gel A (circles), Gel B (squares) and Gel C (diamonds), solid lines correspond to the fit of the curve (see description).

feature in the real part of the dielectric permittivity is transformed into a peak (narrower than the one present in the imaginary part of the dielectric permittivity and without the influence of the dc conductivity). In the figure the two relaxation processes NR (leading to the peak about $10^{2} \mathrm{~Hz}$ ) and DCR (responsible for the peak at $10^{4} \mathrm{~Hz}$ ) are clearly evident for the three samples. In this kind of representation we can follow both relaxation processes down to temperatures of about $350 \mathrm{~K}$. It is quite obvious that the two relaxation processes are faster for Gel B sample. As we are interested in quantifying the differences between the network-related relaxations of the three gels, the analysis of the relaxation characteristics was performed by fitting the derivative data at relatively high temperatures where the $\alpha$-relaxation is out of the window. Thus, the superposition of two symmetric Cole-Cole equations [15] (to take into account DCR and NR processes) plus an extra power law contribution to account for the electrode and/or interfacial polarization phenomena was used,

$$
\epsilon^{\prime}(\omega)=\epsilon_{\infty}+\operatorname{Re}\left(\frac{\Delta \epsilon_{1}}{1+\left(i \omega \tau_{1}\right)^{\alpha_{1}}}+\frac{\Delta \epsilon_{2}}{1+\left(i \omega \tau_{2}\right)^{\alpha_{2}}}\right)+\left(\frac{A}{\omega}\right)^{\kappa} .
$$


Here, $\omega=2 \pi \mathrm{f}, \epsilon_{\infty}$ is the high frequency limiting permittivity, $A$ and $\kappa$ are the parameters used to take into account the electrode and/or interfacial polarization contributions, $\alpha_{i}$ denotes the symmetric broadening of the relaxation component $i(=1,2), \Delta \epsilon_{i}$ corresponds to its relaxation strength and $\tau_{i}$ refers to its characteristic relaxation time.

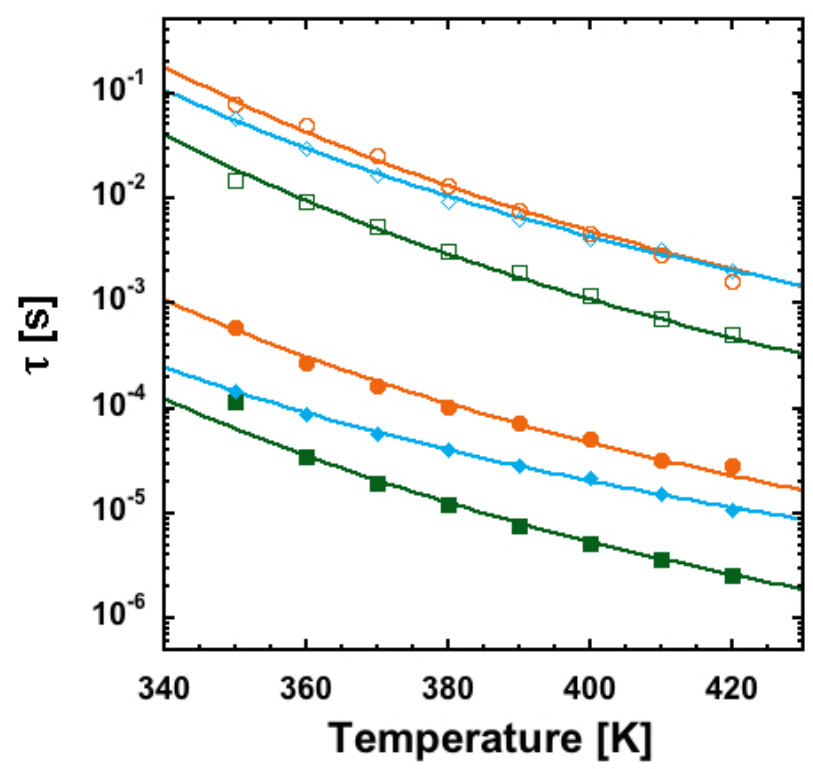

Figure 4: Characteristic relaxation times of the network relaxation (unfilled) and dangling chain relaxation (filled) processes for Gel A (circles), Gel B (squares) and Gel C (diamonds) samples. Lines in the figure correspond to VFT fits.

Figure 4 shows the so obtained characteristic times $\tau$ of both relaxation process (DCR as solid symbols and NR as empty symbols) for the three samples. Both relaxation processes exhibit typical super-Arrhenius temperature dependence. We can notice that in the network relaxation process (empty symbols) Gel A and Gel C exhibit similar characteristic relaxation times around $400 \mathrm{~K}$, however the temperature dependencies are different. Comparing the samples Gel A and Gel B, the opposite situation is found, that is, the temperature dependences are rather similar but the characteristic relaxation times are quite different. In an analogous way, in the case of the dangling chain relaxation process, when comparing the temperature dependence we see that it is quite similar between Gel A and Gel B samples but 
clearly different for Gel C. Furthermore, the relaxation times of the DCR process are quite different for the three samples. Gel B gives the smallest values of the relaxation times, i.e., the fastest relaxation process, followed by Gel $\mathrm{C}$ that gives intermediate values, and Gel A that corresponds to the slowest DCR process. The data in Figure 4 have been described by using a Vogel-Fulcher-Tamman (VFT) equation [16, 17, 18],

$$
\tau=\tau_{\infty} \exp \left(\frac{B}{T-T_{0}}\right)
$$

where $\tau_{\infty}$ is the high temperature limiting relaxation time, $B$ is an energetic constant and $T_{0}$ is the so called Vogel temperature. Due to the relative narrow range of time scales available for each relaxation process, the accurate determination of the three parameters of the VFT by a free fitting is not feasible. Thus, we fixed the value of $T_{0}$ to that determined previously for the $\alpha$-relaxation time of Gel B $\left(T_{0}=158 \mathrm{~K}\right)[10]$. Since, as commented above, the segmental dynamics is not sensitive to the distinct network characteristics of our samples, using the same value of $T_{0}$ for all samples is well justified. Moreover, due to the aforementioned similarities in the temperature dependence between Gel B and Gel A samples we looked for a common $B$ parameter value, on the contrary a different $B$ value was necessary for describing data of Gel C. The so obtained fitting curves are shown as solid lines in Figure 4, being the parameters $\tau_{\infty}$ and $B$ reported in Table 2.

Table 2: Characteristic dielectric relaxation parameters for the gels.

\begin{tabular}{|c|c|c|c|c|c|c|}
\hline Samples & $\tau_{\infty D C R}[\mathrm{~ns}]$ & $B_{D C R}[\mathrm{~K}]$ & $\Delta \epsilon_{D C R}$ & $\tau_{\infty N R}[\mathrm{~ns}]$ & $B_{N R}[\mathrm{~K}]$ & $\Delta \epsilon_{N R}$ \\
\hline Gel A & 3.5 & 2300 & 0.83 & 85 & 2650 & 2.34 \\
\hline Gel B & 0.4 & 2300 & 0.58 & 19 & 2650 & $2.86^{*}$ \\
\hline Gel C & 10.5 & 1830 & 0.65 & 250 & 2360 & 0.88 \\
\hline
\end{tabular}

* The value of the dielectric strength for the network relaxation process in Gel B sample has been recalculated as we detected a mistake in that previously reported. This more accurate value does not change in any sense the discussion and interpretation previously reported.

Now, centering our attention in the relaxation strength (see Figure 5) we notice that the behavior of the three gels is also quite different. The network relaxation process of Gel B presents the largest relaxation strength, decreasing smoothly as the temperature increases. The temperature-dependance 
for the other two samples is similar, yet the relaxation strength of Gel A is much larger than that of Gel C but still smaller than the value of Gel B. For the case of the dangling chain relaxation, the relaxation strengths of Gel $\mathrm{A}, \mathrm{B}$ and $\mathrm{C}$ are quite low. The average relaxation strength values for both relaxation processes at temperatures of around $390 \mathrm{~K}$ are given in Table 2.

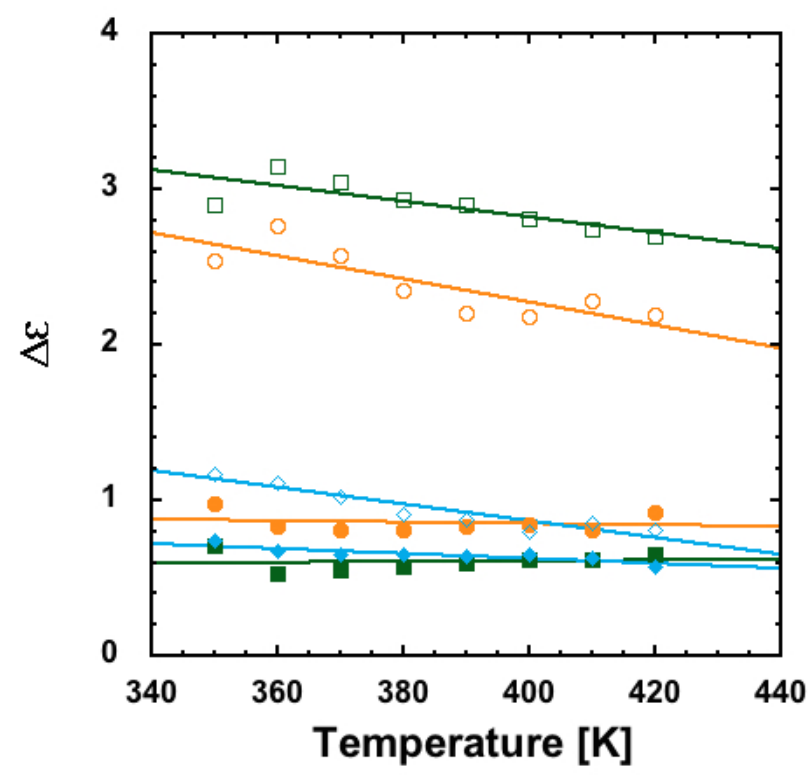

Figure 5: Relaxation strengths of the network relaxation (unfilled) and dangling chain relaxation (filled) processes for Gel A (circles), Gel B (squares) and Gel C (diamonds) samples. Lines in the figure correspond to the linear fit.

\subsection{Small Angle X-ray Scattering}

In order to get insight into the structural features of these materials, we performed X-Ray diffraction experiments covering the SAXS and WAXS regions. The diffractograms for the three gel samples are shown in Figure 6. All gels exhibit two broad but well resolved peaks for high values of the scattering vector, namely, centered around $q_{1} \approx 0.41 \AA^{-1}$ and $q_{2} \approx 1.57 \AA^{-1}$. From these peak positions, periodicity lengths of about 1.5 and $0.4 \mathrm{~nm}$ respectively can be deduced in the Bragg approximation $\left(d=2 \pi / q_{\max }\right)$. These peaks are also present in the reference acrylic sample, as can be seen in Figure 6. A number of structural investigations on polymers with long or bulky side groups $[19,20,21,22,23,24,25]$-like those considered in this work- 
including e. g. the family of poly(n-alkyl acrylates) [19] have established a scenario of nano-phase segregation between main chains and side groups to explain the origin of these peaks in the diffraction patterns [19, 26, 27]. All these systems display a 'pre-peak' in the region where we find $q_{1}$, that has been attributed to the existence of nano-domains rich in each of the polymeric subspecies (main chains or side groups). Such hypothesis was supported by coarse-grained simulations on generic comb-like polymers [28]. Peak at $q_{1}$ would be related to inter-domain correlations [28]. Within this context, peak at $q_{2}$ is assigned to correlations between atoms belonging to side groups of different monomers within a nano-domain.

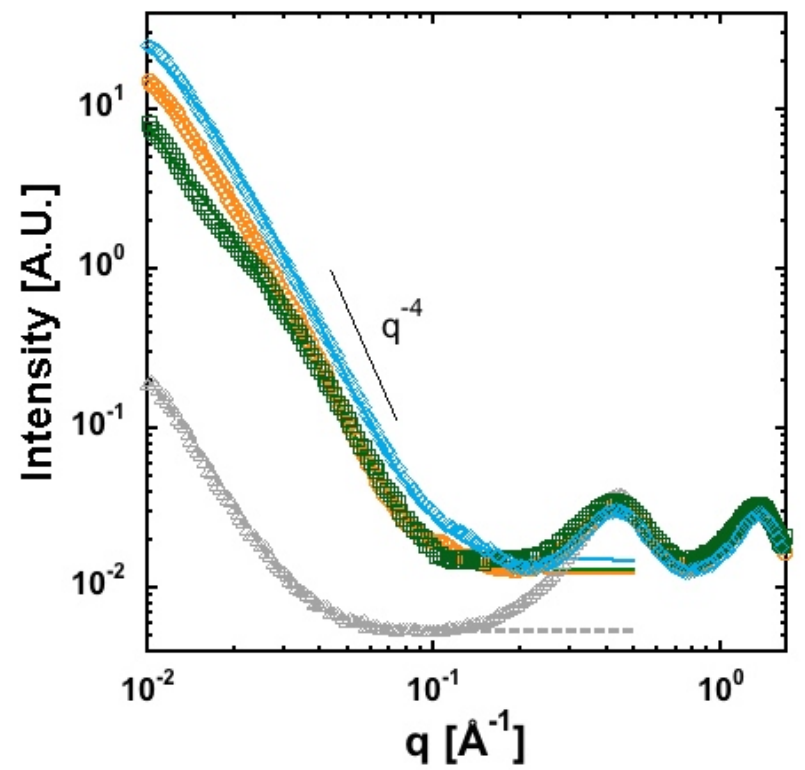

Figure 6: X-Ray diffractograms for Gel A (circles), Gel B (squares) and Gel C (diamonds) at room temperature. Data obtained for the acrylic polymer (triangles) is also shown for comparison. The Lines correspond to different fittings (see the text).

For q-values within the range $\approx 0.1-0.2 \AA^{-1}$, extra intensity contributions are observed for samples that have PU chains incorporated within the synthesis method, i.e., the gel fraction of the Acrylic-PU latex but not the reference linear Acrylic polymer. This extra intensity has to be related with contributions that arise from the contrast given by the presence of PU chains. Further decreasing the q-value, the scattered intensity rapidly increases for the three gel samples. Gels A and C display a slight convexity, while for Gel 
B a broad shoulder is readily resolved. However, the reference linear acrylic polymer also presents a low-q increase of intensity. This behavior can be attributed to the microstructure generated by miniemulsion polymerization, with typical sizes of $100-200 \mathrm{~nm}$ [9]. We have found that this low-q scattered intensity can be well described by a power law with exponent value of -3.6 (see dashed line in Figure 6). Such a scattered intensity is expected to be present in the gel samples also prepared by miniemulsion polymerization but the increasing at low $q$ in the gel samples is more pronounced. The extra low-q intensity in the gel samples at $q \leq 0.2 \AA^{-1}$ can be described by the Debye-Bueche function [29],

$$
I_{D B}=\frac{I_{o}}{\left[1+(\Xi q)^{2}\right]^{2}} .
$$

Where $I_{o}$ is an amplitude factor that depends on the correlation length $\Xi$ and the scattering contrast between the phases leading to the observed inhomogeneities. This function is usually used for neutral gels, to describe the large-scale inhomogeneities produced by concentration fluctuations of the network that are influenced by the physical constraints imposed by the cross-links $[30,31]$. In the present hybrid gels the origin could also be related with the presence of clusters formed by the urethane groups. The fit of the low-q data using Eq. 3 plus a constant background and an additional $q^{-3.6}$ contribution -reflecting the latex structure- is shown by solid lines in Figure 6 . The fitting of Gels $\mathrm{A}$ and $\mathrm{C}$ delivers very similar values for the correlation length ( $\Xi=96$ and $88 \AA$ for Gel A and Gel C, respectively). The intensity $I_{o}$ is somewhat larger for Gel $\mathrm{C}$ than for Gel A. We note the markedly different behavior of Gel B, with a well distinguishable shoulder in the region of about $\approx 0.02 \AA^{-1}$. The value of the correlation length, $\Xi$, obtained for Gel B was $52 \AA$, i.e., it is about half of those observed for Gels A and C.

\section{Discussion}

Our results evidence that the distinct network characteristics of the three gel samples investigated manifest in the dielectric relaxation behavior at frequencies/temperatures lower/higher than that characteristic of the segmental relaxation. However, the segmental dynamics, and the related thermal glasstransition as well, is rather insensitive to the network characteristics. In 
agreement with these results the X-ray diffraction features of the three samples differ mainly at low q values (correlation lengths larger than few $\mathrm{nm}$ ). In the following we discuss the connection of the analysis of the dielectric relaxation data at relatively high temperatures with the structural features determined by SAXS.

In order to understand the differences exhibited by the network relaxation it is useful to recall the molecular interpretation obtained for the dielectric relaxation of these polymers. It was previously reported [10] that Acrylic-PU samples present mainly three different dielectric relaxations, the $\alpha$-relaxation, the dangling chain relaxation and the network relaxation. The dipolar entities responsible for the $\alpha$-relaxation were attributed to segments of the acrylic main chains. In the case of the dangling chain relaxation, the entities were related to PU chains attached by only one end to the acrylic main chain, without being involved in the network formation. Finally, the network relaxation process was reported to be originated by the presence of orientationally correlated urethane-segment bundles belonging to PU chains linked by both ends to the acrylic main chain and forming the gel network. The presence of such large molecular entities responsible for the relaxation phenomena was inferred by the high values of both the dielectric strength and the limiting high temperature relaxation time [10].

Now, when comparing the values of $\tau_{\max }$ obtained for the network relaxation in the three gel samples at $400 \mathrm{~K}$, we see that Gel A and Gel C show both values close to $5 \mathrm{~ms}$ and that $\tau_{\max }$ is nearly a factor of 5 smaller for Gel B (see Figure 4). Thus one would infer that the size of the bundles in Gel B is smaller than that in the other gel samples. This result is well correlated with the SAXS diffraction patterns, where relatively large correlation lengths were inferred for Gel A and Gel C but the correlation length in Gel B was clearly smaller. This direct correspondence between dielectric relaxation results and SAXS can be rationalized considering that the identified SAXS features correspond to the typical distance among bundles. In this case the bigger bundles of Gels $\mathrm{A}$ and $\mathrm{C}$ will be, in average, apart from each other more than the smaller bundles in Gel B, since the PU-Acrylic ratio is quite similar in the three gel samples, being however the value of Gel B slightly lower than those of Gel C and Gel A - the differences of the PU-Acrylic ratio for different samples were estimated using ATR-FTIR measurements, by calculating the ratio of the intensities of two specific PU $\left(1115 \mathrm{~cm}^{-1}\right)$ and Acrylic $\left(1160 \mathrm{~cm}^{-1}\right)$ bands identified in the IR spectra of each Gel sample. 
Regarding the dielectric strength of the network relaxation, we know that this parameter is not only determined by the amount of molecular dipoles participating in the relaxation process but also by any orientational correlation between urethane dipoles constituting these dipolar entities (bundles). Particularly, the larger the orientational correlation between urethane dipoles is, the higher the resulting dipole moment of the bundle entity would be. Since there are no significant differences among the gels in the amount of urethane units, the results obtained for the network relaxation strengths of the different gels (see Table 2) suggest that the correlation between neighboring urethane dipoles forming the bundles is strong in Gel B, not so much in Gel A, and clearly less important in Gel C. The lesser dipolar correlations in Gel $\mathrm{C}$ may be related with the fact that this gel was synthesized by thermal polymerization, whereas Gel B and Gel A both showing significant PU dipole correlation were obtained via photo-polymerization. The different polymerization methods used in the synthesis of the gel samples could also explain the different temperature dependence of the relaxation times manifested in the distinct values of the energetic parameter B obtained by the VFT fitting of both NR and DCR relaxation times (see Table 2). Now the question is what are the reasons for the differences observed between Gel A and Gel B samples, particularly the quite different bundle sizes.

A way of rationalizing the large differences in size and mobility between both gels could be related with constrains imposed by the anchorage of the PU chains to the acrylic-backbone. The shorter the linkage between the bundles and the acrylic backbone is the slower the relaxation is expected to be. Both samples were prepared with the same molecular weight PUprepolymer and with similar concentration of their components (in particular PU $10 \%$ wbo). However, in the synthesis formulation there was also a small fraction of Bisphenol A that promotes the chain extension of the PU chains forming the network by connecting two or more PU pre-polymer chains. Therefore, the present results can be indicative that chain extension occurred more efficiently in Gel B than in Gel A.

To understand how this can occur let us recall some ideas underlying the polymerization process of the produced latexes. Miniemulsion polymerization is one of the most useful techniques for producing waterborne nanocomposites; it allows the possibility of working with pre-formed hydrophobic polymers, plus the advantage that step-growth and free radical polymerization can be carried out within the miniemulsion droplet. In order to obtain hybrid latex products, the polymerization of the acrylic components is 
driven by the free radical polymerization mechanism. On the other hand, the polymerization of the urethane/urea components is activated by step-growth polymerization. Moreover, in the case of miniemulsion photo-polymerization the gels are formed only by reaction of $\mathrm{OH}$ groups of the HEMA units of the acrylic polymer chains (free radical polymerization) with the NCO groups of the PU (step-growth polymerization). The free PU can be incorporated into the acrylic network in one of several distinct ways $[32] ; 1)$ by direct reaction with pendant hydroxyl groups in the acrylate chain, 2) chain extension by Diol?s and subsequent reaction with pendant NCO groups from the acrylic chain, or 3) by addition of water to NCO groups and subsequent rapid urea forming with the pendant NCO groups in the acrylic chain. In the polymerization conditions used for our samples, PU extension seems to be more favorable by reaction with water [32].

The effect of having extended PU chains would be two-fold. On one hand, a larger linkage to the acrylic backbones would reduce the restriction in movement of the bundles related with the proximity of the anchorage site, thus explaining a faster relaxation in Gel B sample. On the other hand, larger linkages would allow a better dispersion of the urethane groups in Gel B sample, which would result in smaller bundles. If this interpretation is correct one should expect a significant amount of chain extender entities in the network of Gel B. Looking for a signature of such extender entities we performed FTIR experiments on Gel A and Gel B samples.

By analyzing both gel samples by FTIR experiments we detected (see Figure 7) extra weak absorption bands for Gel B in the range of 1700 $1500 \mathrm{~cm}^{-1}$ that could be attributed to the presence of these extender entities. When the same comparison is made in samples not previously subjected to a careful drying, also extra absorption bands for Gel B arise in the range of $3300-3500 \mathrm{~cm}^{-1}$ were the $\mathrm{OH}$ related contributions usually appear $[33,34$, 35]. A termogravimetric analysis of the two gels showed that both also differ in the water-vapor uptake ability, up to $3 \%$ for Gel B and less than $0.1 \%$ for Gel A (see the following Figure). The greater water-vapour uptake of Gel B sample could be also rationalized as the effect of the efficient incorporation of chain extenders that would promote the formation of hydrophilic sites. 


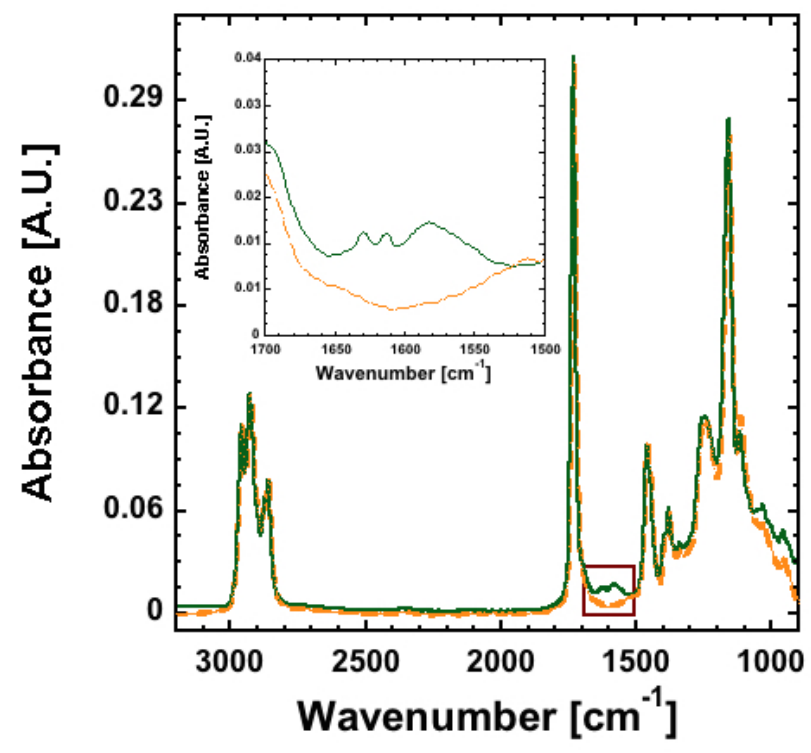

Figure 7: Fourier transform infrared spectrum (FTIR) for Gel A (dashed line) and Gel B (solid line). Inset in the spectrum highlights the differences exhibited by relevant bands in the corresponding range for both samples.

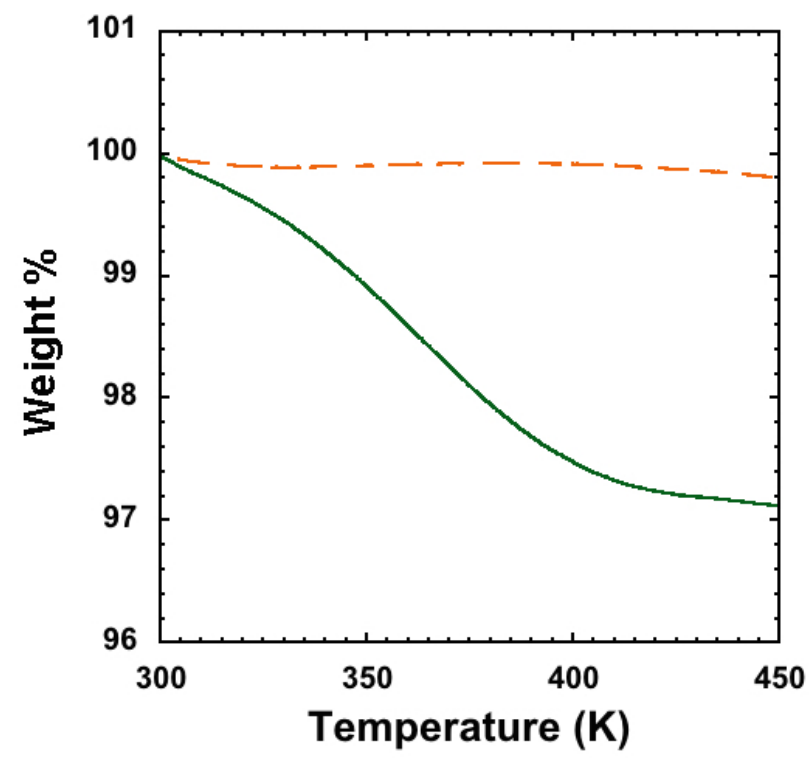

Figure 8: Termogravimetric scans for Gel A (dashed line) and Gel B (solid line) samples. 


\section{Conclusions}

Broadband Dielectric Spectroscopy (BDS) allowed us to identify three different relaxation processes that are characteristic for the gel fraction of Acrylic-PU latexes. Moreover, despite the similar thermal behavior of the three samples, BDS results show up important differences of the characteristic dielectric parameters for two of the relaxation processes detected at frequencies lower than those of the segmental dynamics. Particularly, the network dielectric relaxation process shows differences in the dielectric parameters that have been rationalized in connection with the corresponding synthesis method used. In this way, we show that the gel produced via thermal polymerization presents larger bundles but with poorer orientational correlation among the constituting PU dipoles. Contrary the gel samples obtained via photo-polymerization (Gel A and Gel B) present similar characteristics concerning both, the temperature dependence of the characteristic relaxation times, and the orientational correlation of the constituting $\mathrm{PU}$ dipoles. However, the network relaxation rate of Gel B is about three times faster than that of Gel A, which has been attributed to the significant incorporation of PU chain extenders in Gel B. This finding was supported by FTIR analysis and it can also be the reason for the higher water-uptake tendency of Gel B.

\section{Acknowledgements}

The authors gratefully acknowledge the financial support of the Basque Government project codes: IT-654-13 and nanoIKER2011 - 2013 (Etortek

program) and also the Spanish Ministerio de Economía y competitividad and EU, project codes: MAT2012 - 31088 and MAT2015 - 63704-P.

\section{References}

[1] Benedek I. Pressure-sensitive adhesives and applications. CRC Press; 2004.

[2] Creton C. Pressure-sensitive adhesives: an introductory course. MRS bulletin 2003;28(06):434-9.

[3] Pizzi A, Mittal KL. Handbook of adhesive technology, revised and expanded. CRC press; 2003. 
[4] Udagama R, Degrandi-Contraires E, Creton C, Graillat C, McKenna TF, Bourgeat-Lami E. Synthesis of acrylic- polyurethane hybrid latexes by miniemulsion polymerization and their pressure-sensitive adhesive applications. Macromolecules 2011;44(8):2632-42.

[5] Lopez A, Degrandi-Contraires E, Canetta E, Creton C, Keddie JL, Asua JM. Waterborne polyurethane- acrylic hybrid nanoparticles by miniemulsion polymerization: Applications in pressure-sensitive adhesives. Langmuir 2011;27(7):3878-88.

[6] Asua JM. Miniemulsion polymerization. Progress in polymer science $2002 ; 27(7): 1283-346$.

[7] Degrandi-Contraires E, Udagama R, Bourgeat-Lami E, McKenna T, Ouzineb K, Creton C. Mechanical properties of adhesive films obtained from pu- acrylic hybrid particles. Macromolecules 2011;44(8):2643-52.

[8] Misbah S. Synthesis and characterization of polyurethane acrylate copolymers. Ph.D. thesis; University of Agriculture, Faisalabad; 2012.

[9] Reyes Y, Lopez A, Asua JM. Modeling the microstructure of acrylicpolyurethane hybrid polymers synthesized by miniemulsion polymerization. Macromolecular Reaction Engineering 2011;5(9-10):352-60.

[10] Martínez-Rugerio G, Alegría A, Daniloska V, Tomovska R, Paulis M, Colmenero J. Dielectric relaxations of acrylic-polyurethane hybrid materials. Polymer 2015;74:21-9.

[11] Daniloska V, Tomovska R, Asua JM. Hybrid miniemulsion photopolymerization in a continuous tubular reactor: A way to expand the characteristics of polyurethane/acrylics. Chemical Engineering Journal 2012;184:308-14.

[12] Daniloska V, Carretero P, Tomovska R, Asua JM. High performance pressure sensitive adhesives by miniemulsion photopolymerization in a continuous tubular reactor. Polymer 2014;55(20):5050-6.

[13] Daniloska V, Carretero P, Tomovska R, Paulis M, Asua JM. Highperformance adhesives resulting from spontaneous formation of nanogels within miniemulsion particles. ACS applied materials \& interfaces 2014;6(5):3559-67. 
[14] Wübbenhorst M, van Turnhout J. Analysis of complex dielectric spectra. i. one-dimensional derivative techniques and three-dimensional modelling. Journal of Non-Crystalline Solids 2002;305(1):40-9.

[15] Schönhals A, Kremer F. Analysis of dielectric spectra. In: Broadband dielectric spectroscopy. Springer; 2003, p. 59-98.

[16] Vogel H. The law of the relation between the viscosity of liquids and the temperature. Phys Z 1921;22:645-6.

[17] Fulcher GS. Analysis of recent measurements of the viscosity of glasses. Journal of the American Ceramic Society 1925;8(6):339-55.

[18] Tammann G, Hesse W. Die abhängigkeit der viscosität von der temperatur bie unterkühlten flüssigkeiten. Zeitschrift für anorganische und allgemeine Chemie 1926;156(1):245-57.

[19] Beiner M, Huth H. Nanophase separation and hindered glass transition in side-chain polymers. Nature materials 2003;2(9):595-9.

[20] Genix AC, Lauprêtre F. Subglass and glass transitions of poly (di-nalkylitaconate) s with various side-chain lengths: dielectric relaxation investigation. Macromolecules 2005;38(7):2786-94.

[21] Iradi I, Alvarez F, Colmenero J, Arbe A. Structure factors in polystyrene: a neutron scattering and md-simulation study. Physica B: Condensed Matter 2004;350(1):E881-4.

[22] Arbe A, Genix AC, Colmenero J, Richter D, Fouquet P. Anomalous relaxation of self-assembled alkyl nanodomains in high-order poly (nalkyl methacrylates). Soft Matter 2008;4(9):1792-5.

[23] Gerstl C, Brodeck M, Schneider G, Su Y, Allgaier J, Arbe A, et al. Short and intermediate range order in poly (alkylene oxide) s. a neutron diffraction and molecular dynamics simulation study. Macromolecules 2012;45(17):7293-303.

[24] Busselez R, Arbe A, Alvarez F, Colmenero J, Frick B. Study of the structure and dynamics of poly (vinyl pyrrolidone) by molecular dynamics simulations validated by quasielastic neutron scattering and x-ray diffraction experiments. The Journal of chemical physics 2011;134(5). 
[25] Tejero R, Arbe A, Fernández-García M, López D. Nanostructuration by self-assembly in n-alkyl thiazolium and triazolium side-chain polymethacrylates. Macromolecules 2015;48(19):7180-93.

[26] Wind M, Graf R, Renker S, Spiess HW, Steffen W. Structure of amorphous poly-(ethylmethacrylate): A wide-angle x-ray scattering study. The Journal of chemical physics 2005;122(1).

[27] Hiller S, Pascui O, Budde H, Kabisch O, Reichert D, Beiner M. Nanophase separation in side chain polymers: new evidence from structure and dynamics. New Journal of Physics 2004;6(1):10.

[28] Moreno AJ, Arbe A, Colmenero J. Structure and dynamics of selfassembled comb copolymers: comparison between simulations of a generic model and neutron scattering experiments. Macromolecules 2011;44(6):1695-706.

[29] Debye P, Bueche A. Scattering by an inhomogeneous solid. Journal of Applied Physics 1949;20(6):518-25.

[30] Geissler E, Horkay F, Hecht AM. Scattering from network polydispersity in polymer gels. Physical review letters 1993;71(4):645.

[31] Horkay F, Basser PJ, Hecht AM, Geissler E. Structural investigations of a neutralized polyelectrolyte gel and an associating neutral hydrogel. Polymer 2005;46(12):4242-7.

[32] Ballard N, Carretero P, Asua JM. Effect of reaction temperature on adhesive properties of waterborne polyurethane/acrylic hybrids synthesized by semicontinuous miniemulsion polymerization. Macromolecular Reaction Engineering 2013;7(10):504-14.

[33] Delpech MC, Miranda GS. Waterborne polyurethanes: influence of chain extender in ftir spectra profiles. Central European Journal of Engineering 2012;2(2):231-8.

[34] Peruzzo PJ, Anbinder PS, Pardini OR, Costa CA, Leite CA, Galembeck F, et al. Polyurethane/acrylate hybrids: effects of the acrylic content and thermal treatment on the polymer properties. Journal of applied polymer science 2010;116(5):2694-705. 
[35] Peruzzo PJ, Anbinder PS, Pardini OR, Vega JR, Amalvy JI. Influence of diisocyanate structure on the morphology and properties of waterborne polyurethane-acrylates. Polymer journal 2012;44(3):232-9. 